\title{
Palisaded neutrophilic and granulomatous dermatitis - cutaneous manifestation of Lyme disease or connected with CTD? Case report
}

\author{
Magdalena Pirowska', Aleksander Obtułowicz', Grzegorz Dyduch², Sylwia Lipko-Godlewska', \\ Anna Wojas-Pelc ${ }^{1}$ \\ ${ }^{1}$ Department of Dermatology, Jagiellonian University Medical College, Cracow, Poland \\ 2 Department of Pathomorphology, Jagiellonian University Medical College, Department of Pathomorphology, \\ Jagiellonian University Medical College, Cracow, Poland
}

Pirowska M, Obtułowicz A, Dyduch G, Lipko-Godlewska S, Wojas-Pelc A. Palisaded neutrophilic and granulomatous dermatitis - cutaneous manifestation of Lyme disease or connected with CTD? Case report. Ann Agric Environ Med. 2016; 23(2): 384-386. doi: 10.5604/12321966.1203913

\begin{abstract}
Introduction. Palisaded neutrophilic and granulomatous dermatitis (PNGD) are terms which include such diseases as rheumatoid nodules, Churg-Strauss granuloma, and interstitial granulomatous dermatitis with arthritis. This heterogeneous group was first described in 1965. It is associated with immunological diseases. There are reported cases of coexistence with systemic lupus erythematosus, rheumatoid arthritis, Wegener's granuloma, inflammatory bowel disease, generalized vascular inflammation, and lymphoproliferative disorders. The etiology of the disease is unknown. It is probable that the deposition of immune complexes in blood vessels leads to cutaneus leukocytoclastic vasculitis, degeneration of collagen fibres and palisaded granulomatous inflammation. Ultimately, this leads to fibrosis of the skin.

Objective. The aim of the study is to present a patient with skin lesions and histopathological features of palisaded neutrophilic and granulomatous dermatitis during the course of infection Borrelia burgdorferi. Several cases of this disease have been reported worldwide (30 entries in the PubMed database). To-date, there have been no reports of PNGD in Polish literature.

Materials and method. The patient, aged 72, was admitted to hospital because of erythematous, indurated lesion of the skin on the side surface of the left thigh. Diagnosis of PNGD was made on the basis of typical histopathologic features due to clinical symptoms.

Conclusions. Clinical diagnosis of PNGD is difficult, and is based mainly on the histopathological picture. Systemic therapy is incorporated mainly due to the systemic disease. The patient requires further observation in the direction of associated systemic disorders.
\end{abstract}

\section{Key words}

Palisaded neutrophilic and granulomatous dermatitis, borreliosis (Lyme disease), lupus erythematosus

\section{INTRODUCTION}

Palisaded neutrophilic and granulomatous dermatitis (PNGD) is a rare entity that has not been clearly defined, nither histopathologically nor clinically [1]. The first cases of PNGD were described in 1965 by Dykman et al., who presented two patients with skin lesions on the lateral trunk that resembled indurated linear and arcuate cords or bands, associated with rheumatoid arthritis [2]. Histopathological examination revealed an interstitial granulomatous dermatitis with lymphocytes and neutrophils, as well as areas of degenerated collagen. Nowadays, PNGD is an umbrella term used to encompass non-specific skin lesions due to such disorders as rheumatoid papules, interstitial granulomatous dermatitis with arthritis (Ackerman syndrome), and Churg-Strauss disease [3]. A PubMed search identified only 21 publications about palisaded neutrophilic and granulomatous dermatitis. No cases of this disease have been described previously in Poland.

\footnotetext{
Address for correspondence: Magdalena Pirowska, Department of Dermatology, Jagiellonian University Medical College, ul. Gołębia 24, 31-007 Cracow, Poland E-mail: pirowska@gmail.com

Received: 17 July 2013; accepted: 06 May 2014
}

\section{CASE REPORT}

A 71-year-old woman presented with a 4-month history of tender, smooth, erythematous plaques on the lower extremities and trunk. Physical examination revealed multiple 'woody hard'infiltrative lesions, erythematous-toviolaceus in colour. Most of the lesions were medium in size, measuring approximately $3-5 \mathrm{~cm}$ in diameter. The first lesion appeard on the left hip; surface of this plaque was rough and covered with exfoliating epidermis. A similar lesion was localized above the left knee (Fig. 1). On the trunk, there were many smaller lesions, likewise hard and firm, but the skin above the lesions was not so inflamed as the previous one (Fig. 2). The patient had no lymphadenopathy.

The eruptions did not disappeared spontaneously and new lesions appeared intermittently. She complained of diffuse myalgia and occasional fatigue with a slight fever of $37.5-37.8^{\circ} \mathrm{C}$. Review of systems including morning stiffness of phalangeal joints. No history of oral ulcers or alopecia was elicited.

Past medical history included myocardial ischaemia, hypertension, diabetes mellitus type 2 and hypercholesterolaemia.

Laboratory examinations showed sightnormochromic normocytic anaemia (Hb 10.92 g/dL, Ht 32.02\%, MCV 


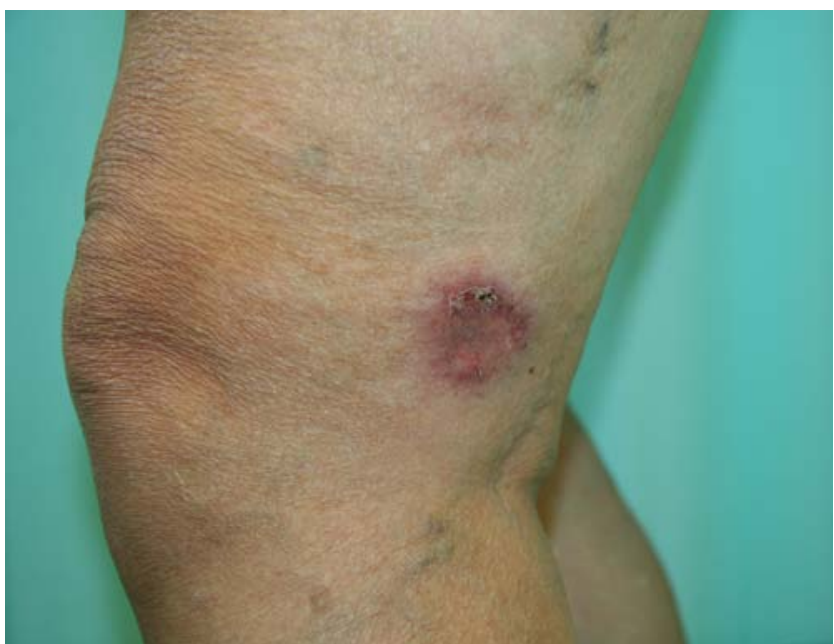

Figure 1. Plaque localized above the left knee

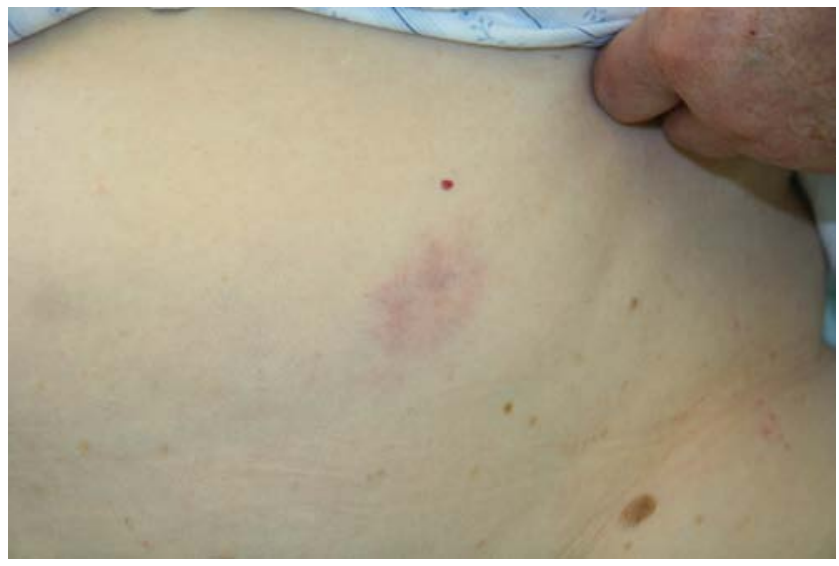

Figure 2. Smaller lesion on the trunk

$88.6 \mathrm{fL}, \mathrm{MCH} 30.2 \mathrm{pg}$ ) with signs of inflammation (ASR 23/56mm qand CRP $12.4 \mathrm{mg} / \mathrm{L}$ ). Antinuclear antibody titer was 1:320 with a homogenous pattern. Anti-Ro/La, antidouble-stranded DNA, anti-cardiolipin, anti-CCP, and antiSCL-70 antibodies were negative. A complete blood count, basic metabolic panel, hepatic function tests, iron level tests (TIBC, UIBC, ferritin and transferrin), C3 and C4 levels, and urinalysis were normal. Hepatitis $\mathrm{B}$ antigen, $\mathrm{C}$ antibody and tumour markers detected by immunohistochemistry were negative. Serological tests for Lyme disease revealed positive reactions in the serum for IgG antibodies $(>30 \mathrm{BBU} / \mathrm{ml})$ and negative for $\operatorname{IgM}$ antibodies $(0.7 \mathrm{BBU} / \mathrm{ml})$. Photo test (exposure to UVB) was positive. Minimal dose that caused erythema in 24 hours was $0.038 \mathrm{~J} / \mathrm{cm} 2$. Punch biopsy was performed to aid the diagnosis of the eruption. Within the superficial and deep dermis there was a perivascular interstitial mixed-cell infiltrate, composed of neutrophils, histiocytes and lymphocytes. In some foci, the inflammatory infiltrate was palisaded and associated with basophilic alteration of collagen (Fig. 3). These findings were interpreted as PNGD. Narrow linear IgG and C3c staining of the basement membrane was seen in direct immunofluorescence (Fig. 4). The patient was treated with cefuroxin $2 \times 500 \mathrm{mg}$ p.o., topical methyprednizoloni aceponas was also prescribed.

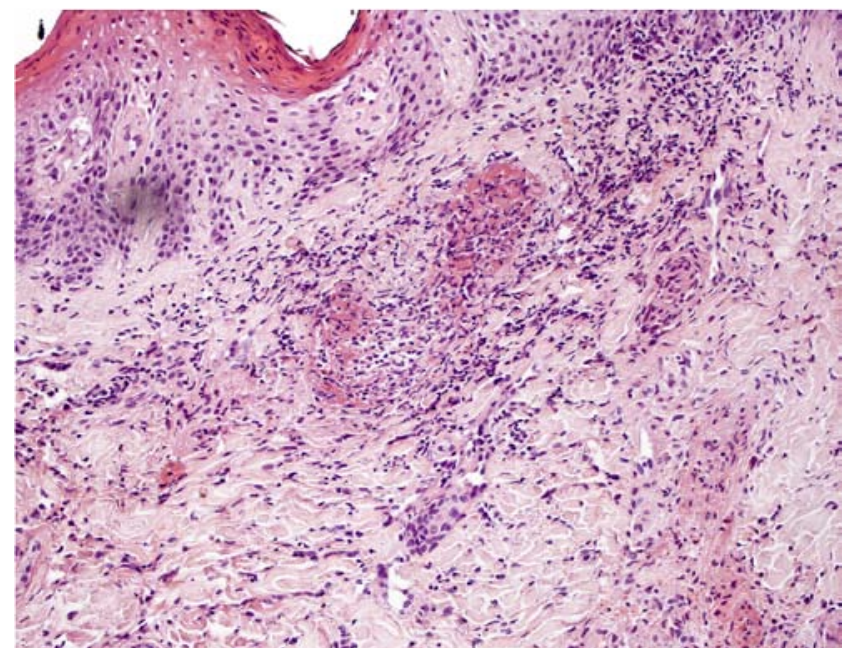

Figure 3. Palisaded inflamatory infiltrate (histopathology of the lesion)

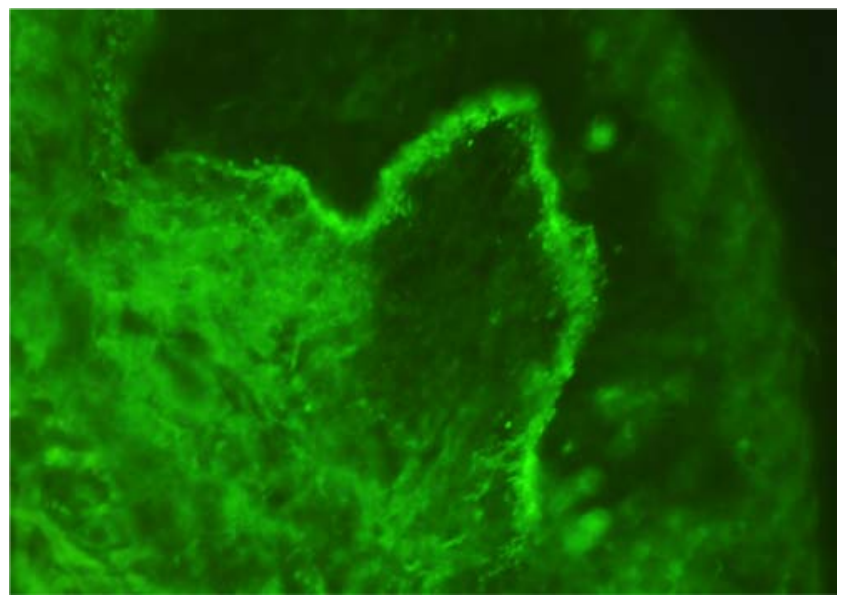

Figure 4. Linear IgG and C3c staining of the basement membrane in direct immunofluorescence

\section{DISCUSSION}

Chu et al. defined palisaded neutrophilic granulomatous dermatitis in 1994 as a disease in which circulating immune complexes occur and can develop a papular eruption on the extremities [4]. The authors suggest that PNGD represents a continuum with an inciting lesion representing leukocytoclastic vasculitis, and with palisaded granulomas observed in fully developed lesions [5]. The term 'palisaded', when used in dermopathology, refers to the arrangement of histiocytes in an inflammatory infiltrate [2]. The lesions of palisaded neutrophilic and granulomatous dermatitis are usually symmetrically distributed on the extremities and have smooth, ulcerated, or umbilicated surfaces [6]. However, PNGD can present as pink to violaceus papules, plaques, nodules or linear bands, some of which can have urticarial or annular configurations [5]. Early lesions are urticaria-like annular plaques, or may have a livedoid appearance. In later stages, the lesions are more infiltrative and pleomorphic (e.g. violaceous annular plaques, waxy papules, painful subcutaneous nodules, indurated linear bands) [5].

Finally, in the presented case, fibrosis was observed. The overlying epidermis is usually normal, although sometimes crusts and ulcer appear. Linear subcutaneous indurated 
cordlike bands, coined the 'rope sign', first described in 1993 by Ackerman, are pathognomonic of interstitial granulomatous dermatitis [7]. The duration of the disease in the presented case was not long, but the lesions were more advanced than observed in another cases.

PNDG is heterogenous entity associated with systemus lupus erythematosus, rheumatoid arthritis, Wegener's granulomatosis, inflamatory bowel disease, and lymphoproliferative disease $[3,8]$. Single cases of Behcet disease, hepatitis, sarcoidosis, leukocytoclastic vasculitis, systemic sclerosis has been reported $[9,10,11,12,13]$. Recently, two groups of scientist, Newell et al. and Misago et al., reported cases of patients with PNGD with no underlying illness $[11,14,15]$. The patient in the current case presented 2 of 11 SLE symptoms (ACR): photosensitivity, positive ANA, and additionaly, linear IgG andC $3 \mathrm{c}$ staining of the basement membrane in direct immunofluorescence. She had positive IgG antibodies against Borrelia burgorferi, through which Lyme disease was recognized.

The etiology of PNGD is unknown, although in 1983, Finan and Winkelmann, using direct immunofluorescent examination, observed $\operatorname{IgM}$ and $\mathrm{C} 3$ in small vessels 16]. They presumed that the cutaneous lesions were the result of immune complexes generated by underlying systemic diseases [16]. Similar observations were seen in the presented case, there was narrow linear IgG and C3c staining of the basement membrane.

Differential diagnoses include interstitial granuloma annulare, leucocytoclastic vasculitis, urticarial vasculitis, Sweet syndrome, rheumatoid nodules, necrobiosis lipoidica, and erythema elevatum et diutinum $[1,8]$. Most eruptions are asymptomatic, but our patient complained tenderness. Sometimes pruritus appear [8].

Various treatment modalities tried include oral steroids, dapsone, colchicine, cyclosporine, cyclophosphamide, hydroxychloroquine, and nonsteroidal anti-inflamatory agents, with variable success rates. Oral steroids appear to be the most beneficial therapy $[1,17]$. Brechter tried to treat individual lesions with intralesional glucocorticoid injections, with some improvement [6]. Many cases have not responded to therapy and reccurences were common after treatment with prednisone was tapered $[6,7]$. The condition may persist from several months to a few years [8]. Usually, the clinical course of PNGD appears to be self-limitted, but this was not observe in the presented patient. Whether the appearance of PNGD in an autoimmune disease is associated with a worse therapeutic rensponse, or a poor prognosis, has not yet been clarified [11].

Due to Al-Daraji et al., the authors of the presented case consider that the lack of silent diagnostic features, both clinically and pathologically, a high index of suspicion is mandatory for making the correct diagnosis [3].

\section{REFERENCES}

1. Gutte R, Khopkar U. Isolated unilateral palisaded neutrophilic and granulomatous dermatitis.Indian J Dermatol Venereol Leprol. 2011; 77(5): 615-7.

2. Bremner R, Simpson E, White CR, Morrison L, Deodhar A. Palisaded neutrophilic and granulomatous dermatitis: an unusual cutaneous manifestation of immune-mediated disorders. Semin Arthritis Rheum. 2004; 34(3): 610-6.

3. Al-Daraji WI, Coulson IH, Howat AJ. Palisaded neutrophilic and granulomatous dermatitis. Clin Exp Dermatol. 2005; 30(5): 578-9.

4. Chu P, Connolly MK, LeBoit PE. The histopathologic spectrum of palisaded neutrophilic and granulomatous dermatitis in patients with collagen vascular disease. Arch Dermatol. 1994; 130(10): 1278-83.

5. Stephenson SR, Campbell SM, Drew GS, Magro CM. Palisaded neutrophilic and granulomatous dermatitis presenting in a patient with rheumatoid arthritis on adalimumab. J Cutan Pathol. 2011; 38(8): 644-8.

6. Brecher A. Palisaded neutrophilic and granulomatous dermatitis. Dermatol Online J. 2003; 9(4): 1.

7. Gulati A, Paige D, Yaqoob M, Proby CM, Cerio R, Harwood CA. Palisaded neutrophilic granulomatous dermatitis associated with systemic lupus erythematosus presenting with the burning rope sign. J Am Acad Dermatol. 2009; 61(4): 711-4.

8. Heidary N, Mengden S, Pomeranz MK. Palisaded neutrophilic and granulomatous dermatosis. Dermatol Online J. 2008 May 15;14(5): 17.

9. Szepetiuk G, Lesuisse M, Piérard GE, Quatresooz P, PiérardFranchimont C.Autoimmunity-related granulomatous dermatitis in association with hepatitis.Case Rep Dermatol. 2012; 4(1): 80-4.

10. Mahmoodi M, Ahmad A, Bansal C, Cusack CA.Palisaded neutrophilic and granulomatous dermatitis in association with sarcoidosis. J Cutan Pathol. 2011; 38(4): 365-8.

11. Misago N, Shinoda Y, Tago M, Narisawa Y. Palisaded neutrophilic granulomatous dermatitis with leukocytoclastic vasculitis in a patient without any underlying systemic disease detected to date. J Cutan Pathol. 2010; 37(10): 1092-7.

12. Asahina A, Fujita H, Fukunaga Y, Kenmochi Y, Ikenaka T, Mitomi H. Early lesion of palisaded neutrophilic granulomatous dermatitis in ulcerative colitis.Eur J Dermatol. 2007; 17(3):234-7.

13. Kim SK, Park CK, Park YW, Jun JB, Yoo DH, Bae SC. Palisaded neutrophilic granulomatous dermatitis presenting as an unusual skin manifestation in a patient with Behçet's disease. Scand J Rheumatol. 2005; 34(4): 324-7.

14. Fett N, Kovarik C, Bennett D. Palisaded neutrophilic granulomatous dermatitis without a definable underlying disorder treated with dapsone. J Am Acad Dermatol. 2011; 65(3): e92-3.

15. Newell EL, Mallipeddi R, Murdoch ME, Groves R, Black MM, Robson A. A case of cutaneous extravascular necrotizing granuloma without systemic manifestations. Clin Exp Dermatol. 2007; 32(5): 509-12.

16. Chou CL, Wang KH, Chiang YY. Palisaded neutrophilic and granulomatous dermatitis associated with the initiation of etanercept in rheumatoid arthritis: a case report. Dermatol Sin. 2011;29(4): 129-133.

17. Sangueza OP, Caudell MD, Mengesha YM, Davis LS, Barnes CJ, Griffin JE et al. Palisaded neutrophilic granulomatous dermatitis in rheumatoid arthritis. J Am Acad Dermatol. 2002; 47(2): 251-7. 